\title{
10-Jahres-Ergebnisse: Sichere Behandlung mit Radiofrequenzablation
}

Hintergrund und Fragestellung: Grundsätzlich kann neben der etablierten chirurgischen Metastasenresektion auch die Radiofrequenzablation zur lokalen Destruktion von Lebermetastasen kolorektaler Karzinome mit Aussicht auf langfristige komplette Remission eingesetzt werden, eventuell auch in Kombination mit der chirurgischen Therapie. Mit der vorliegenden Arbeit sollten die Langzeitergebnisse dieses Verfahrens überprüft werden.

Patienten und Methoden: Die Autoren behandelten von 1997 bis 2006 konsekutiv 99 Patienten mit insgesamt 202 metachronen Metastasen kolorektaler Karzinome (Durchmesser 0,8 bis $4,0 \mathrm{~cm}$; Median 2,2 cm) mit einer durch Ultraschall gesteuerten, perkutanen Radiofrequenzablation (RFA). Die Patienten waren entweder für eine chirurgische Resektion ungeeignet $(\mathrm{n}=80)$ oder lehnten die

Originalie

Solbiati L et al. Small liver colorectal metastases treated with percutaneous radiofrequency ablation: local response rate and long-term survival with up to 10-year follow-up. Radiology. 2012;265(3):958-68
Operation ab. Offenbar erhielten alle Patienten zusätzlich eine systemische Chemotherapie. Nach der RFA wurden die Patienten computertomografisch mit Kontrastmittelverstärkung oder mit Magnetresonanztomografie (MRT) über einen Zeitraum von mindestens
3 Jahren bzw. bis zu mehr als 10 Jahre nachverfolgt. Zielkriterien waren die lokale Kontrolle und Überlebensraten. Kaplan-MeierKurven wurden generiert und Log-rank-Tests durchgeführt.

Ergebnisse: Die primäre und sekundäre technische Erfolgsrate lag jeweils bei $93,1 \%(188 / 202)$ und $100 \%(14 / 14)$. Insgesamt $11,9 \%$ aller behandelten Metastasen (24/202) waren progredient. Etwas mehr als die Hälfte dieser Metastasen (54,2\%) wurden erneut behandelt. Das Patientenüberleben verbesserte sich durch die wiederholte Behandlung gegenüber keiner Rezidivtherapie signifikant $(\mathrm{p}<0,001)$. Im Verlauf wurden 125 neue Lebermetastasen entdeckt und 41 von ihnen mit RFA behandelt $(32,8 \%)$. Das Gesamtüberleben (OS) betrug nach 1, 3, 5, 7 und 10 Jahren jeweils $98 \%, 69,3 \%, 47,8 \%, 25 \%$ und $18 \%$ (Median 53,2 Monate). Schwerwiegende Komplikationen traten bei zwei Patienten auf, jedoch ohne letalen Ausgang.

Schlussfolgerung der Autoren: Zusammen mit der systemischen Chemotherapie erreicht die RFA bei einer großen Mehrheit der Patienten mit kleinen Lebermetastasen kolorektaler Karzinome eine lokale Kontrolle. Die Überlebensraten nach 3 und 10 Jahren entsprechen jenen in den meisten chirurgischen Serien.

Schlüsselwörter: Kolorektales Karzinom - Lebermetastasen Radiofrequenzablation

\section{- Kommentar von Werner Hohenberger, Erlangen}

\section{„Die Schlussfolgerung der Autoren muss eingeschränkt werden“}

Das Ergebnis der Arbeit von Solbiati und Kollegen reiht sich in eine ganze Reihe weiterer Publikationen zur Radiofrequenzablation von Lebermetastasen kolorektaler Karzinome ein. Die Schlussfolgerung der Autoren, dass nämlich damit gezeigt wird, dass mit der Radiofrequenzablation gleichwertige Langzeitergebnisse wie mit der chirurgischen Resektion zu erreichen sind, muss jedoch eingeschränkt werden. Zum einen handelt es sich bei dem dargestellten Krankengut um Patienten mit metachronen Metastasen, die letztendlich eine bessere Prognose haben als solche mit synchronen Metastasen. Zum anderen betrug der mediane Metastasendurchmesser nur 2,2 cm (0,8-4,0 cm). Und nur für diese kleinen Metastasen mit einem Durchmesser bis $z u \quad 3 \mathrm{~cm}$ sind die Behandlungsergebnisse der Radiofrequenzablation vergleichbar mit denen der chirurgischen Resektion. Allerdings liegen hierzu keine Daten aus randomisierten Studien vor. Diesbezüglich wurden zwar verschiedene Versuche unternommen, die aber scheiterten.

\section{Fazit}

Die Radiofrequenzablation kann zur Behandlung von Lebermetastasen kolorektaler Karzinome herangezogen werden, die im Durchmesser $\leq 3 \mathrm{~cm}$ sind. Größere Metastasen, die im Gesunden entfernbar sind, werden nach wie vor chirurgisch reseziert.

Erstpubliziert in Strahlenther Onkol. 2013;189(5):430

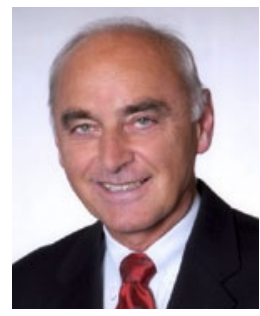

Prof. Dr. Dr. Werner Hohenberger

Chirurgische Universitätsklinik

Erlangen

werner.hohenberger@uk-erlangen.de 